\title{
Impact of Nursing Intervention on Urostomy Patients Outcome Regarding Practicing Self-Care
}

\author{
Zienab Abd El-Latif Mohamed*, Amal Mohamed Ahmad*
}

\begin{abstract}
Urinary diversion operations (urostomy) patients require a great deal of emotional and physical adjustment. They usually face many problems such as loss of body function and relationship with others, and change in lifestyle. They need to adapt to the external appliance, stoma alteration in toileting habits, skin irritation problems, infection, and odor problems. Selfcare for these patients is considered the most important factor that can minimize the complaints and complications. The aim of this study was to evaluate the impact of nursing intervention among urostomy patients regarding practicing self-care. The sample comprised 100 urostomy patients, divided into two groups: 50 study, and 50 control. For both groups, patients' knowledge about urostomy and related self-care performance were assessed before intervention, immediately after, and after six-months follow-up at outpatient clinic. Data were analyzed using Epi-info 6.04 computer software package. Findings revealed statistically significant improvements in knowledge and performance among patients in the study group, compared to the control group. The study group became more knowledgeable about the definition, function, anatomy, and physiology of urostomy, and in self-care performance. The rates of complaints and complications were found to be lower in the study group than in the control one. The study suggests that urostomy patients should receive their self-care training regarding urostomy during hospitalization. Follow-up at outpatient clinic is highly recommended as well.
\end{abstract}

\section{INTRODUCTION}

Bladder cancer is one of the most common diseases treated by the urologists. It is the second most commonly occurring genito-urinary cancer in adults. In men, it is the fourth most common cancer after prostate, lung, and colorectal cancers, while in females bladder cancer is the eighth most common type of cancers $^{(1-3)}$.

In Egypt, there is a high incidence of bladder cancer. It is generally categorized into bilharzial and nonbilharzial types. Bilharzial type is prevalent in rural areas, and affects adult males. These patients usually present for

*Medical-Surgical Nursing, Faculty of Nursing, Assiut University 
treatment at an advanced stage of the lifestyle. They need to adapt to the disease, and its pathology is commonly external appliance, stoma, alteration in squamous cell carcinoma. On the other toileting habits, and urinary drainage, in hand, non-bilharzial bladder cancer is addition to skin irritation problems, observed among elderly patients who urinary tract infections, and odor may present for treatment with $\operatorname{problems}^{(8,9) \text {. }}$ transitional cell carcinoma ${ }^{(4,5)}$.

Therefore, permanent urostomy

The word urostomy signifies a type of surgery required when a person has permanent or temporary loss of the normal function of the bladder or bowel due to birth defects, cancer, injury, or other diseases ${ }^{(6)}$. The surgery allows for normal bodily wastes to be expelled through a new surgical opening urostomy on the abdominal wall. Most persons with ostomies wear special appliances over the stoma(7).

Following urinary diversion operations, patients require a great deal of emotional and physical adjustment. They usually face many problems such as loss of body functions and relationship with others, altered sexuality, and change in means long-term change in an individual's lifestyle and compliance with new patterns of behavior requiring a life long process of self-care. Various studies have shown that nearly half of long-term urostomy patients do not comply with self-care, such as diet, accepting stoma, and doing irrigation(10). Recently, there has been a growing realization and awareness of the importance of patient self-care concerning their surgical ostomy on discharge from hospital(11).

Self-care for urostomy is considered the most important factor that can minimize the complaints and complications rate, as well as providing 
good health status. These patients have important tasks and responsibilities through hospitalization and at home. They should be taught how to manage and minimize the complications ${ }^{(12)}$.

Nurses play a vital role regarding cystectomized patients. This roleincludes monitoring acute reaction, follow-up care of these patients, and providing education, counseling and support as the patient faces urinary diversion ${ }^{(13)}$. Also nurses play an important role to help these patients to cope and adapt to their illness. For this, nurses have to develop intervention programs that take the form of psychoeducational preparation aimed at helping the patients to develop acceptance of self despite the disability, to enhance their knowledge for effective coping strategies, and to increase their selfesteem and sense of control $(8,14)$.

\section{Significance of the study}

From clinical experience and observation of the actual situation, it was obvious to the researchers that urostomy patients and their family members need to know as much as possible about this treatment modality, and the related selfcare. To the best knowledge of the researchers, some studies were done in Egypt for assessment of nurses' knowledge and nursing interventions for postoperative urology and abdominal surgeries. However, no studies were done on self-care for urinary diversion patients.

\section{Study aim:}

The aim of this study was to:

1. Assess urostomy patients' knowledge and self-care practices related to urostomy before implementation of nursing intervention.

2. Evaluate the effect of nursing intervention on urostomy patients' outcome in practicing self-care.

\section{Research hypotheses}


1. Urostomy patients who received the nursing intervention will be more knowledgeable about self-care, compared to control ones

2. Urostomy patients who received the training program will have significantly better practice of selfcare, compared to control group who did not receive nursing intervention.

\section{MATERIAL AND METHODS}

\section{Research design}

A quasi-expermental design was used in the conduction of this study, with comparison of the outcomes between study and control groups immediately after nursing intervention and at 6-month follow-up.

\section{Setting}

The study was conducted at the Urology Department (inpatients and outpatients) at Assiut University Hospital

\section{Subjects}

A sample of 100 patients attending the study setting and fulfilling the inclusion and exclusion criteria was consecutively recruited. The inclusion criteria were being adult patient with urostomy done at the study setting and during the time of the study. The only exclusion criterion was the presence of concomitant chronic diseases such as diabetes mellitus, etc. The total sample was randomized into two equal groups of 50 patients each, (the study and control groups).

\section{Tools}

Three tools were developed by the researchers to be used for data collection.

\section{1- Urostomy Patient assessment}

sheet: This tool was an Arabic semistructured interview questionnaire sheet constructed by the researchers after reviewing related literature $\mathrm{e}^{(15-17)}$. The tool consisted of two parts. The first part included questions about the socio-demographic characteristics of 
the patients. The second part was intended to assess patient' knowledge about the urostomy such as related basic anatomy and physiology, definition and reason of urostomy performance, function, as well as urostomy self-care. For the knowledge items, a correct response was scored 1 and the incorrect zero. For each part, the scores of the items were summed-up and the total was divided by the number of the items, giving a mean score for the part. These scores were converted into a percent score. The patient knowledge was considered satisfactory if the percent score was $50 \%$ or more, and unsatisfactory if less than $50 \%$.

11 - Urostomy Self-care performance checklist: This was developed by the researchers after reviewing the related literature ${ }^{(18-21)}$. This tool included three parts: Part one, to assess patients' performance of urostomy self-care immediately after the nursing intervention before discharge, and at 6month follow-up. It includes skin care around the urostomy, care of the appliance, preparation, removal and irrigation and fixation. Part two, it includes recording any complications during the follow-up period. For scoring the observation checklists, the items "not done" and "done" were scored "0"and "1", respectively. For each part, the scores of the items were summedup and the total was divided by the number of the items, giving a mean score for the part. These scores were converted into a percent score. The practice was considered adequate if the percent score was $60 \%$ or more, and inadequate if less than $60 \%$. Part three, to assess patients' attitude towards the urostomy and its appliance. It involved statements related to care of urostomy, daily life activities with urostomy, as well as acceptance of the stoma. For 
scoring, the responses "agree", patient's practice related to appliance "uncertain", and "disagree" were technique, and how to handle respectively scored 3, 2, and 1 .The complications. Besides, the researchers scoring was reversed for negative provided health education regarding diet, statements. The scores of the items bowel, irrigation, early complications, were summed-up and the total was sexual relations, clothing, and divided by the number of the items, employment.

giving a mean score. These scores were converted into a percent score. The attitude was considered positive if the percent score was $60 \%$ or more, and negative if less than $60 \%$.

\section{Methods}

1. This study was conducted through a period of six months from October 2005 to March 2006.

2. 5 senior faculty experts in the field judged the questionnaire, and self

\section{The nursing intervention:}

A self-care practicing was developed based on patients' needs assessment The content of the nursing intervention was developed after reviewing related literature ${ }^{(17,22-27)}$. The prepared material was then translated into Arabic. The knowledge topics in the nursing intervention covered the reason for urostomy performance, definition, basic anatomy and physiology, self-care,

3. Obtaining the official permission to conduct the study, as well as the consents from patients to ensure their willingness to engage in the study after explaining its purpose and nature.

4. Nursing intervention design and planning, with the objectives, teaching methods, and media to be used. 
5. A pilot study after development of the data collection tools. This was carried out on ten patients to test tools feasibility and applicability.

6. Implement the nursing intervention for the study group members. This was in the form of a two-hour session per day. The session started with theoretical background followed by practical information. The total number of sessions was five, totaling to ten hours for the nursing intervention. Different teaching/learning activities were used during individual training, including discussion, demonstration, and redemonstration. Real urostomy appliances were used for training. Handouts such as booklets, pamphlets, and posters were also prepared and used by researchers. For the control group, hospital care and procedures were applied. They were practicing their own self-care according to available doctors and nurses' instructions.
7. Estimation of the effect of the nursing intervention on practicing self-care. This was done immediately after the intervention implemented and six months later.

8. Patients in both groups were asked to come to the outpatient department to assess their self-care performance, and to record any complications. The visits were also used to provide a booster dose of health education to the study group members.

9. The effectiveness of nursing intervention was evaluated using the same tools in both groups at discharge and at the 6-month follow-up visit.

\section{Statistical analysis}

Data analysis was done using Epi-Info 6.04 computer software package. Data were presented using descriptive statistics in the form of frequencies and percentages. Qualitative variables were compared using chi-square test. Whenever the expected values in one or more of the cells in a $2 \times 2$ tables was less than 5, Fisher exact test was used instead. In larger than $2 \times 2$ cross-tables, 
no test could be applied whenever the expected value in $10 \%$ or more of the cells was less than 5. Statistical significance was considered at $p$-value $<0.05$.

\section{RESULTS}

Table 1 describes the socio-demographic characteristics of urostomy patients in the study and control groups. About three-fifths of the patients in both groups were below the age of 40 years. They were mostly males,
$64.0 \%$ and $68.0 \%$, respectively. The highest percentages were illiterate or could only read and write, $64.0 \%$ and $56.0 \%$, respectively, and were married, $72.0 \%$ and $84.0 \%$, respectively. Almost all of the patients in the two groups were working. As the table demonstrates, no differences of statistical significance could be found between the two groups

Table 1. Socio-demographic characteristics of urostomy patients in both the study and control groups

\begin{tabular}{|c|c|c|c|c|c|c|}
\hline & \multicolumn{2}{|c|}{$\begin{array}{l}\text { Study group } \\
\text { No. } 50\end{array}$} & \multicolumn{2}{|c|}{$\begin{array}{c}\text { Control group } \\
\text { No. } 50\end{array}$} & \multirow{2}{*}{$\chi^{2}$} & \multirow{2}{*}{$p$-value } \\
\hline & No & $\%$ & & & & \\
\hline \multicolumn{7}{|l|}{ Age: } \\
\hline$<30$ & 10 & 20.0 & 16 & 32.0 & & \\
\hline $30-$ & 20 & 40.0 & 14 & 28.0 & & \\
\hline $40-$ & 12 & 24.0 & 10 & 20.0 & & \\
\hline $50+$ & 8 & 16.0 & 10 & 20.0 & 2.85 & 0.42 \\
\hline \multicolumn{7}{|l|}{ Gender: } \\
\hline Male & 32 & 64.0 & 34 & 68.0 & & \\
\hline Female & 18 & 36.0 & 16 & 32.0 & 0.18 & 0.67 \\
\hline \multicolumn{7}{|l|}{ Education level: } \\
\hline Illiterate/read-write & 32 & 64.0 & 28 & 56.0 & & \\
\hline Basic education & 12 & 24.0 & 10 & 20.0 & & \\
\hline Secondary/university & 6 & 12.0 & 12 & 24.0 & 2.45 & 0.29 \\
\hline \multicolumn{7}{|l|}{ Marital status: } \\
\hline Single & 12 & 24.0 & 8 & 16.0 & & \\
\hline Married & 36 & 72.0 & 42 & 84.0 & & \\
\hline Divorced /Widowed & 2 & 4.0 & 0 & 0.0 & -- & -- \\
\hline \multicolumn{7}{|l|}{ Occupation: } \\
\hline Working & 46 & 92.0 & 50 & 100.0 & & \\
\hline Not working & 4 & 8.0 & 0 & 0.0 & Fisher & 0.12 \\
\hline
\end{tabular}

\section{(--) Test result not valid}

A comparison of the preintervention knowledge about urostomy between study and control group patients is illustrated in table 2. It shows 
generally low levels of satisfactory better knowledge regarding definition of knowledge in both groups. Some urostomy, $p=0.02$, patients in the control differences of statistical significance group had better knowledge regarding were revealed between the two groups. care of skin around the stoma, $p=0.004$, While patients in the study group had and stoma self-care, $p=0.004$.

Table 2: Comparison of pre-intervention knowledge related to urostomy between the study and control groups

\begin{tabular}{|c|c|c|c|c|c|}
\hline & Stu & & Cont & & $\chi^{2}$ \\
\hline & No. & $\%$ & No. & $\%$ & \\
\hline $\begin{array}{c}\text { Basic anatomy/physiology: } \\
\text { Satisfactory } \\
\text { Unsatisfactory }\end{array}$ & $\begin{array}{l}15 \\
35 \\
\end{array}$ & $\begin{array}{l}30.0 \\
70.0\end{array}$ & $\begin{array}{l}14 \\
36 \\
\end{array}$ & $\begin{array}{l}28.0 \\
72.0 \\
\end{array}$ & $\begin{array}{l}0.05 \\
0.83\end{array}$ \\
\hline $\begin{array}{c}\text { Definition of urostomy: } \\
\text { Satisfactory } \\
\text { Unsatisfactory }\end{array}$ & $\begin{array}{l}11 \\
39\end{array}$ & $\begin{array}{l}22.0 \\
78.0\end{array}$ & $\begin{array}{r}3 \\
47 \\
\end{array}$ & $\begin{array}{r}6.0 \\
94.0\end{array}$ & $\begin{array}{c}5.32 \\
0.02^{\star}\end{array}$ \\
\hline $\begin{array}{r}\text { Function of urostomy: } \\
\text { Satisfactory } \\
\text { Unsatisfactory }\end{array}$ & $\begin{array}{r}42 \\
8\end{array}$ & $\begin{array}{l}84.0 \\
16.0\end{array}$ & $\begin{array}{r}44 \\
6\end{array}$ & $\begin{array}{l}88.0 \\
12.0\end{array}$ & $\begin{array}{l}0.33 \\
0.56\end{array}$ \\
\hline $\begin{array}{r}\text { Reasons of urostomy: } \\
\text { Satisfactory } \\
\text { Unsatisfactory }\end{array}$ & $\begin{array}{r}3 \\
47 \\
\end{array}$ & $\begin{array}{r}6.0 \\
94.0 \\
\end{array}$ & $\begin{array}{r}9 \\
41 \\
\end{array}$ & $\begin{array}{l}18.0 \\
82.0 \\
\end{array}$ & $\begin{array}{l}3.41 \\
0.07\end{array}$ \\
\hline $\begin{array}{l}\text { Urostomy care: } \\
\text { Satisfactory } \\
\text { Unsatisfactory }\end{array}$ & $\begin{array}{l}10 \\
40 \\
\end{array}$ & $\begin{array}{l}20.0 \\
80.0\end{array}$ & $\begin{array}{l}10 \\
40\end{array}$ & $\begin{array}{l}20.0 \\
80.0\end{array}$ & -- \\
\hline $\begin{array}{c}\text { Appliance care: } \\
\text { Satisfactory } \\
\text { Unsatisfactory }\end{array}$ & $\begin{array}{l}13 \\
37\end{array}$ & $\begin{array}{l}26.0 \\
74.0\end{array}$ & $\begin{array}{l}10 \\
40\end{array}$ & $\begin{array}{l}20.0 \\
80.0\end{array}$ & $\begin{array}{l}0.51 \\
0.48\end{array}$ \\
\hline $\begin{array}{c}\text { Care of skin around stoma: } \\
\text { Satisfactory } \\
\text { Unsatisfactory }\end{array}$ & $\begin{array}{r}2 \\
48 \\
\end{array}$ & $\begin{array}{r}4.0 \\
96.0\end{array}$ & $\begin{array}{l}12 \\
38 \\
\end{array}$ & $\begin{array}{l}24.0 \\
76.0\end{array}$ & $\begin{array}{c}8.31 \\
0.004^{*}\end{array}$ \\
\hline $\begin{array}{c}\text { Stoma self care: } \\
\text { Satisfactory } \\
\text { Unsatisfactory }\end{array}$ & $\begin{array}{r}5 \\
45\end{array}$ & $\begin{array}{l}10.0 \\
90.0\end{array}$ & $\begin{array}{l}17 \\
33\end{array}$ & $\begin{array}{l}34.0 \\
66.0\end{array}$ & $\begin{array}{c}8.39 \\
0.004^{*}\end{array}$ \\
\hline
\end{tabular}

$\left.{ }^{*}\right)$ Statistically significant at $p<0.05$

As regards pre-practice related to and control groups, table 3 also urostomy among patients in the study demonstrates generally low levels of 
adequate practice in both groups. significance could be detected between

However, no differences of statistical the group

Table 3. Comparison of pre-practice related to urostomy between the study and control groups

\begin{tabular}{|c|c|c|c|c|c|}
\hline & \multicolumn{2}{|c|}{$\begin{array}{l}\text { Study } \\
\text { group } \\
(\mathrm{n}=50)\end{array}$} & \multicolumn{2}{|c|}{$\begin{array}{l}\text { Control } \\
\text { group } \\
(\mathrm{n}=50)\end{array}$} & \multirow[t]{2}{*}{$\begin{array}{c}\chi^{2} \\
\mathrm{p}- \\
\text { value }\end{array}$} \\
\hline & No. & $\%$ & No. & $\%$ & \\
\hline $\begin{array}{l}\text { Clean stoma (using soft tissue, proper } \\
\text { appliance, prescribed medication, stoma } \\
\text { irrigation): }\end{array}$ & & & & & \\
\hline Adequate & 13 & 26.0 & 13 & 26.0 & -- \\
\hline Inadequate & 37 & 74.0 & 37 & 74.0 & -- \\
\hline $\begin{array}{l}\text { Report any abnormality (dryness, changes } \\
\text { in stoma color, size, stenosis, edema, } \\
\text { discharge, or leakage): }\end{array}$ & & & & & \\
\hline Adequate & 19 & 38.0 & 19 & 38.0 & -- \\
\hline Inadequate & 31 & 62.0 & 31 & 62.0 & -- \\
\hline $\begin{array}{l}\text { Clean skin around stoma (dry skin using } \\
\text { soap and warm water, apply protective } \\
\text { medication): }\end{array}$ & & & & & \\
\hline Adequate & 22 & 44.0 & 22 & 44.0 & -- \\
\hline Inadequate & 28 & 56.0 & 28 & 56.0 & -- \\
\hline $\begin{array}{l}\text { Care of appliance (preparation, removal, } \\
\text { fixation): }\end{array}$ & & & & & \\
\hline Adequate & 17 & 34.0 & 17 & 34.0 & -- \\
\hline Inadequate & 33 & 66.0 & 33 & 66.0 & -- \\
\hline $\begin{array}{l}\text { Observing urine characteristics (color, } \\
\text { amount, odor, turbidity, content): }\end{array}$ & & & & & \\
\hline Adequate & 19 & 38.0 & 19 & 38.0 & -- \\
\hline Inadequate & 31 & 62.0 & 31 & 62.0 & -- \\
\hline
\end{tabular}

Comparisons of the post-nursing all areas of knowledge $(p<0.001)$, as intervention knowledge about urostomy presented in table 4 . In all these between study and control group patients differences, the percentages of satisfactory immediately and at 6-month follow-up have knowledge were higher among patients in shown statistically significant differences in the study 
Group, compared to the control group. increased from $94.0 \%$ at the immediate At the follow-up, the percentages of test to 100.0 at the six-month follow-up satisfactory knowledge have shown some test. Nonetheless, still the differences declines in most items, although the between study and control groups were of knowledge about stoma self-care statistical significance at the follow-up tes

Table 4. Comparison of knowledge related to immediate and at 6-month follow-up between the study and control groups

\begin{tabular}{|c|c|c|c|c|c|c|c|c|c|c|}
\hline & \multicolumn{4}{|c|}{$\begin{array}{l}\text { Study group } \\
(n=50)\end{array}$} & \multicolumn{4}{|c|}{$\begin{array}{c}\text { Control group } \\
(n=50)\end{array}$} & \multirow{3}{*}{$\begin{array}{l}\text { Immedi } \\
\text { ate } \\
\chi^{2} \\
\text { p-value }\end{array}$} & \multirow{3}{*}{$\begin{array}{l}\text { 6-month } \\
\chi^{2} \\
\text { p-value }\end{array}$} \\
\hline & \multicolumn{2}{|c|}{ Immediate } & \multicolumn{2}{|c|}{ 6-month } & \multicolumn{2}{|c|}{ Immediate } & \multicolumn{2}{|c|}{ 6-month } & & \\
\hline & No. & $\%$ & No. & $\%$ & No. & $\%$ & No. & $\%$ & & \\
\hline $\begin{array}{l}\text { Basic anatomy } \\
\text { /physiology: } \\
\text { Satisfactory } \\
\text { Unsatisfactory }\end{array}$ & $\begin{array}{l}37 \\
13\end{array}$ & $\begin{array}{l}74.0 \\
26.0\end{array}$ & $\begin{array}{l}31 \\
19\end{array}$ & $\begin{array}{l}62.0 \\
38.0\end{array}$ & $\begin{array}{l}10 \\
40\end{array}$ & $\begin{array}{l}20.0 \\
80.0\end{array}$ & $\begin{array}{r}8 \\
42 \\
\end{array}$ & $\begin{array}{l}16.0 \\
84.0\end{array}$ & $\begin{array}{c}29.27 \\
<0.001^{*}\end{array}$ & $\begin{array}{c}32.78 \\
<0.001^{*}\end{array}$ \\
\hline $\begin{array}{c}\text { Definition of urostomy: } \\
\text { Satisfactory } \\
\text { Unsatisfactory }\end{array}$ & $\begin{array}{r}41 \\
9 \\
\end{array}$ & $\begin{array}{l}82.0 \\
18.0 \\
\end{array}$ & $\begin{array}{l}37 \\
13 \\
\end{array}$ & $\begin{array}{l}74.0 \\
26.0 \\
\end{array}$ & $\begin{array}{l}20 \\
30 \\
\end{array}$ & $\begin{array}{l}40.0 \\
60.0 \\
\end{array}$ & $\begin{array}{l}21 \\
29 \\
\end{array}$ & $\begin{array}{l}42.0 \\
58.0 \\
\end{array}$ & $\begin{array}{r}18.54 \\
<0.001^{*} \\
\end{array}$ & $\begin{array}{c}10.51 \\
<0.001^{*} \\
\end{array}$ \\
\hline $\begin{array}{r}\text { Function of urostomy: } \\
\text { Satisfactory } \\
\text { Unsatisfactory }\end{array}$ & $\begin{array}{r}45 \\
5\end{array}$ & $\begin{array}{l}90.0 \\
10.0\end{array}$ & $\begin{array}{l}40 \\
10\end{array}$ & $\begin{array}{l}80.0 \\
20.0\end{array}$ & $\begin{array}{r}5 \\
45 \\
\end{array}$ & $\begin{array}{l}10.0 \\
90.0\end{array}$ & $\begin{array}{r}7 \\
43 \\
\end{array}$ & $\begin{array}{l}14.0 \\
86.0\end{array}$ & $\begin{array}{c}64.00 \\
<0.001^{*}\end{array}$ & $\begin{array}{c}43.72 \\
<0.001^{*}\end{array}$ \\
\hline $\begin{array}{r}\text { Reasons of urostomy: } \\
\text { Satisfactory } \\
\text { Unsatisfactory }\end{array}$ & $\begin{array}{l}39 \\
11\end{array}$ & $\begin{array}{l}78.0 \\
22.0\end{array}$ & $\begin{array}{l}32 \\
18 \\
\end{array}$ & $\begin{array}{l}64.0 \\
36.0\end{array}$ & $\begin{array}{l}25 \\
25\end{array}$ & $\begin{array}{l}50.0 \\
50.0\end{array}$ & $\begin{array}{l}18 \\
32 \\
\end{array}$ & $\begin{array}{l}36.0 \\
64.0\end{array}$ & $\begin{array}{c}8.51 \\
0.004^{*}\end{array}$ & $\begin{array}{c}7.84 \\
0.005^{*}\end{array}$ \\
\hline $\begin{array}{l}\text { Urostomy care: } \\
\text { Satisfactory } \\
\text { Unsatisfactory }\end{array}$ & $\begin{array}{l}36 \\
14 \\
\end{array}$ & $\begin{array}{l}72.0 \\
28.0\end{array}$ & $\begin{array}{l}36 \\
14 \\
\end{array}$ & $\begin{array}{l}72.0 \\
28.0 \\
\end{array}$ & $\begin{array}{l}10 \\
40 \\
\end{array}$ & $\begin{array}{l}20.0 \\
80.0\end{array}$ & $\begin{array}{l}10 \\
40 \\
\end{array}$ & $\begin{array}{l}20.0 \\
80.0\end{array}$ & $\begin{array}{r}27.21 \\
<0.001^{*} \\
\end{array}$ & $\begin{array}{c}27.21 \\
<0.001^{*}\end{array}$ \\
\hline $\begin{array}{c}\text { Appliance care: } \\
\text { Satisfactory } \\
\text { Unsatisfactory }\end{array}$ & $\begin{array}{l}36 \\
14 \\
\end{array}$ & $\begin{array}{l}72.0 \\
28.0 \\
\end{array}$ & $\begin{array}{l}36 \\
14 \\
\end{array}$ & $\begin{array}{l}72.0 \\
28.0 \\
\end{array}$ & $\begin{array}{l}10 \\
40 \\
\end{array}$ & $\begin{array}{l}20.0 \\
80.0 \\
\end{array}$ & $\begin{array}{l}10 \\
40 \\
\end{array}$ & $\begin{array}{l}20.0 \\
80.0 \\
\end{array}$ & $\begin{array}{r}27.21 \\
<0.001^{*} \\
\end{array}$ & $\begin{array}{r}27.21 \\
<0.001^{*} \\
\end{array}$ \\
\hline $\begin{array}{l}\text { Care of skin around } \\
\text { stoma: } \\
\\
\quad \text { Satisfactory } \\
\\
\text { Unsatisfactory }\end{array}$ & $\begin{array}{r}43 \\
7 \\
\end{array}$ & $\begin{array}{l}86.0 \\
14.0 \\
\end{array}$ & $\begin{array}{r}41 \\
9 \\
\end{array}$ & $\begin{array}{l}82.0 \\
18.0 \\
\end{array}$ & $\begin{array}{l}15 \\
35 \\
\end{array}$ & $\begin{array}{l}30.0 \\
70.0 \\
\end{array}$ & $\begin{array}{l}13 \\
37 \\
\end{array}$ & $\begin{array}{l}26.0 \\
74.0 \\
\end{array}$ & $\begin{array}{r}32.18 \\
<0.001^{*} \\
\end{array}$ & $\begin{array}{r}31.56 \\
<0.001^{*} \\
\end{array}$ \\
\hline $\begin{array}{l}\text { Stoma self care: } \\
\text { Satisfactory } \\
\text { Unsatisfactory }\end{array}$ & $\begin{array}{r}47 \\
3 \\
\end{array}$ & $\begin{array}{r}94.0 \\
6.0 \\
\end{array}$ & $\begin{array}{r}50 \\
0 \\
\end{array}$ & $\begin{array}{r}100.0 \\
0.0 \\
\end{array}$ & $\begin{array}{l}12 \\
38 \\
\end{array}$ & $\begin{array}{l}24.0 \\
76.0 \\
\end{array}$ & $\begin{array}{l}11 \\
39 \\
\end{array}$ & $\begin{array}{l}22.0 \\
78.0 \\
\end{array}$ & $\begin{array}{r}50.64 \\
<0.001^{*} \\
\end{array}$ & $\begin{array}{c}63.93 \\
<0.001^{*} \\
\end{array}$ \\
\hline
\end{tabular}

$\left(^{*}\right)$ Statistically significant at $p<0.05$

Table 5 illustrates the comparison of postpractices related to urostomy between study and control group patients immediately and at 6-month follow-up. It is evident that the percentages of adequate practice were higher among patients in the study group, 
compared to the control group at both phases. At the immediate phase, these differences have reached statistical significance in cleaning stoma $(p=0.005)$, reporting any abnormality $(p<0.001)$, and cleaning skin around stoma $(p=0.03)$. At the six-month follow-up, still the differences between study and control groups were of statistical significance in the first two of these three items. Concerning care for appliance and observation of urine, the table shows no differences of statistical significance between the two groups.

Table 5. Comparison of practice related to immediate and at 6-month follow-up between the study and control groups

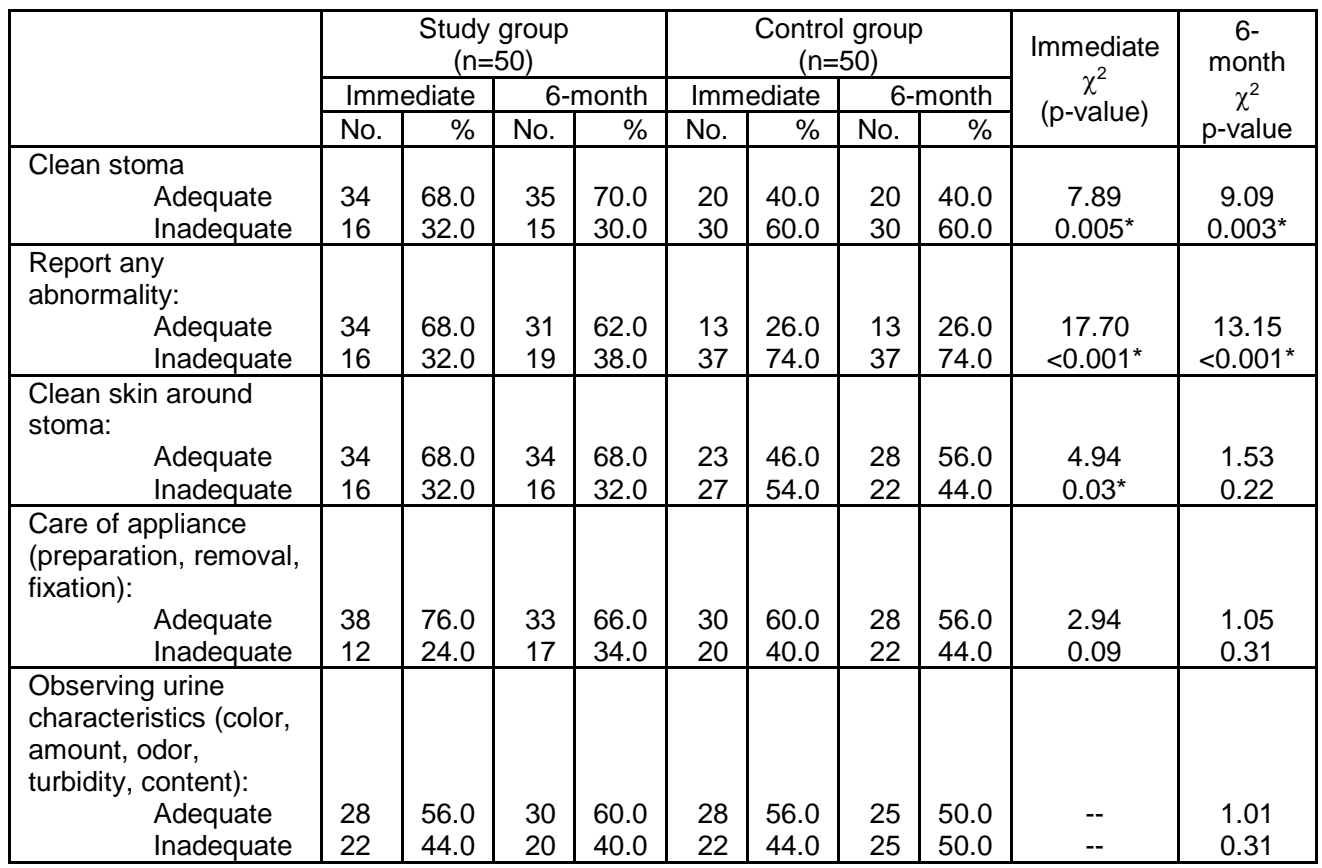

$\left(^{*}\right)$ Statistically significant at $p<0.05$

(--) Test result not valid

immediately and at 6-month follow-up. It is

As regards patients' attitude towards

urostomy, table 6 presents a comparison

noticed that the percentages of positive between study and control patients attitude were higher among patients in the 
study group, compared to the control group at both phases. All these differences were of statistical significance at both phases. The only exception was the attitude towards acceptance of stoma. Although more

Table 6. Comparison of attitudes related to immediate and at 6-month follow-up between urostomy patients in the study and control groups

\begin{tabular}{|c|c|c|c|c|c|c|c|c|c|c|}
\hline & \multicolumn{4}{|c|}{$\begin{array}{c}\text { Study group } \\
(\mathrm{n}=50)\end{array}$} & \multicolumn{4}{|c|}{$\begin{array}{c}\text { Control group } \\
(\mathrm{n}=50)\end{array}$} & \multirow{3}{*}{$\begin{array}{c}\text { Immediate } \\
\chi^{2} \\
\text { (p-value) }\end{array}$} & \multirow{3}{*}{$\begin{array}{c}\text { 6-month } \\
\chi^{2} \\
\text { p-value }\end{array}$} \\
\hline & \multicolumn{2}{|c|}{ Immediate } & \multicolumn{2}{|c|}{ 6-month } & \multicolumn{2}{|c|}{ Immediate } & \multicolumn{2}{|c|}{ 6-month } & & \\
\hline & No. & $\%$ & No. & $\%$ & No. & $\%$ & No. & $\%$ & & \\
\hline $\begin{array}{r}\text { Stoma care: } \\
\text { Positive } \\
\text { Negative } \\
\end{array}$ & $\begin{array}{l}38 \\
12 \\
\end{array}$ & $\begin{array}{l}76.0 \\
24.0\end{array}$ & $\begin{array}{l}35 \\
15 \\
\end{array}$ & $\begin{array}{l}70.0 \\
30.0\end{array}$ & $\begin{array}{l}20 \\
30 \\
\end{array}$ & $\begin{array}{l}40.0 \\
60.0 \\
\end{array}$ & $\begin{array}{l}20 \\
30 \\
\end{array}$ & $\begin{array}{l}40.0 \\
60.0\end{array}$ & $\begin{array}{r}13.30 \\
<0.001^{*} \\
\end{array}$ & $\begin{array}{c}9.09 \\
0.003^{*}\end{array}$ \\
\hline $\begin{array}{r}\text { Appliance care: } \\
\text { Positive } \\
\text { Negative } \\
\end{array}$ & $\begin{array}{l}34 \\
16 \\
\end{array}$ & $\begin{array}{l}68.0 \\
32.0 \\
\end{array}$ & $\begin{array}{l}30 \\
20 \\
\end{array}$ & $\begin{array}{l}60.0 \\
40.0 \\
\end{array}$ & $\begin{array}{l}18 \\
32 \\
\end{array}$ & $\begin{array}{l}36.0 \\
64.0 \\
\end{array}$ & $\begin{array}{l}19 \\
31 \\
\end{array}$ & $\begin{array}{l}38.0 \\
62.0 \\
\end{array}$ & $\begin{array}{l}10.26 \\
0.002^{*}\end{array}$ & $\begin{array}{l}4.84 \\
0.03^{*} \\
\end{array}$ \\
\hline $\begin{array}{r}\text { Daily life activities: } \\
\text { Positive } \\
\text { Negative } \\
\end{array}$ & $\begin{array}{l}41 \\
9 \\
\end{array}$ & $\begin{array}{l}82.0 \\
18.0 \\
\end{array}$ & $\begin{array}{l}39 \\
11 \\
\end{array}$ & $\begin{array}{l}78.0 \\
22.0 \\
\end{array}$ & $\begin{array}{l}22 \\
28 \\
\end{array}$ & $\begin{array}{l}44.0 \\
56.0 \\
\end{array}$ & $\begin{array}{l}22 \\
28 \\
\end{array}$ & $\begin{array}{l}44.0 \\
56.0\end{array}$ & $\begin{aligned} & 15.49 \\
&<0.001^{*} \\
&\end{aligned}$ & $\begin{array}{r}12.15 \\
<0.001^{*} \\
\end{array}$ \\
\hline $\begin{array}{l}\text { Acceptance of } \\
\text { stoma: } \\
\quad \text { Positive } \\
\\
\text { Negative } \\
\end{array}$ & $\begin{array}{l}39 \\
11\end{array}$ & $\begin{array}{l}78.0 \\
22.0\end{array}$ & $\begin{array}{l}36 \\
14\end{array}$ & $\begin{array}{l}72.0 \\
28.0\end{array}$ & $\begin{array}{l}32 \\
18\end{array}$ & $\begin{array}{l}64.0 \\
36.0 \\
\end{array}$ & $\begin{array}{l}31 \\
19\end{array}$ & $\begin{array}{l}62.0 \\
38.0\end{array}$ & $\begin{array}{l}2.38 \\
0.12 \\
\end{array}$ & $\begin{array}{l}1.13 \\
0.29 \\
\end{array}$ \\
\hline
\end{tabular}

$\left({ }^{*}\right)$ Statistically significant at $p<0.05$

Table 7 and Figure 1 demonstrate statistically significant lower percentages of stoma, skin, and psychological problems among patients in the study group, $40.0 \%$, patients in the study group had positive attitude, compared to the control group, the differences were not statistically significant, $p>0.05$. 


\begin{tabular}{|c|c|c|c|c|c|c|}
\hline & No & $\%$ & No & $\%$ & & \\
\hline $\begin{array}{c}\text { Stoma complications: } \\
\text { Yes }\end{array}$ & 20 & 40.0 & 46 & 92.0 & & \\
No & 30 & 60.0 & 4 & 8.0 & 30.12 & $<0.001^{*}$ \\
\hline $\begin{array}{c}\text { Skin complications: } \\
\text { Yes }\end{array}$ & 18 & 36.0 & 44 & 88.0 & & \\
No & 32 & 64.0 & 6 & 12.0 & 28.69 & $<0.001^{*}$ \\
\hline $\begin{array}{c}\text { Psychological disorders: } \\
\text { Yes }\end{array}$ & 24 & 48.0 & 42 & 84.0 & & \\
No & 26 & 52.0 & 8 & 16.0 & 14.44 & $<0.001^{*}$ \\
\hline
\end{tabular}

$\left(^{*}\right)$ Statistically significant at $p<0.05$

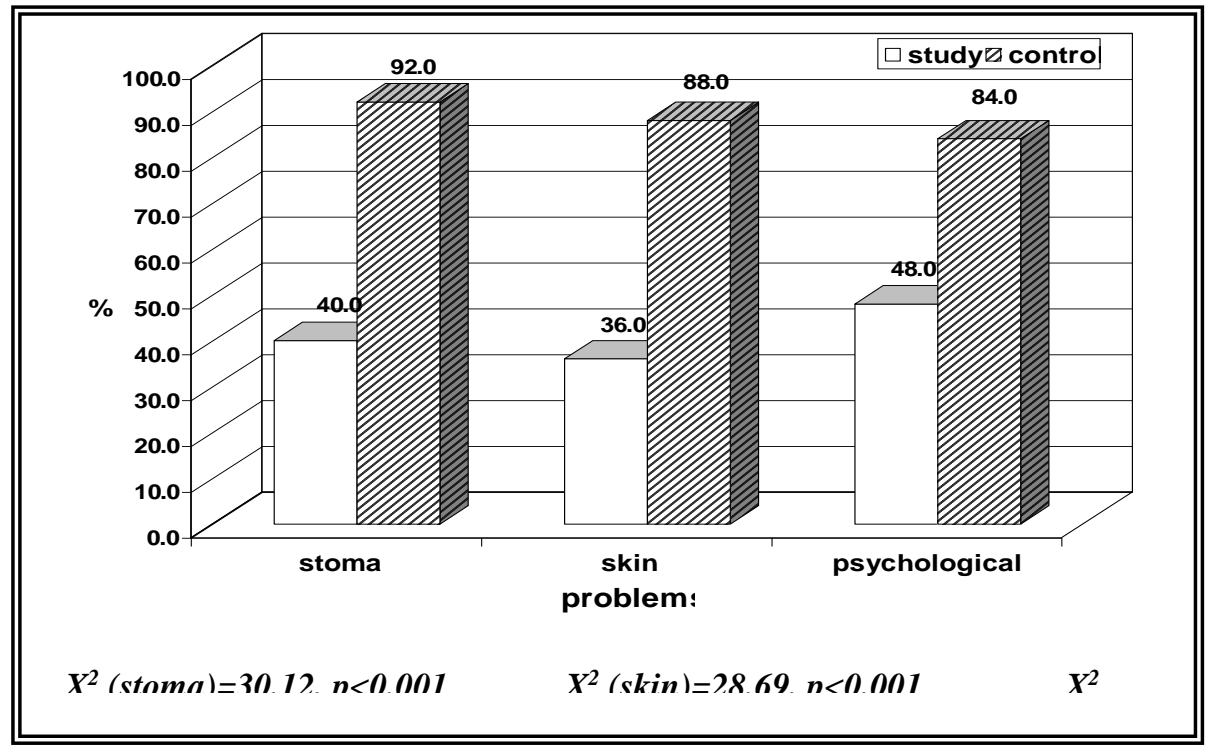

Figure 1. Stoma problems among patients in the study and control groups throughout the intervention

\section{DISCUSSION}

Each year, tens of thousands of people of all ages call for an ostomy as part of the treatment and recovery. An ostomy may be the best and safest form of treatment for a number of conditions such as cancer of the urinary bladder, trauma, malformation present from birth, and obstruction of the urinary bowel(6). Yet, when the physician first mentions an ostomy, every patient without exception has fears and worries about how to adapt to this change(28). Thus, the construction of the urostomy 
can affect patients' psychosocial wellbeing, particularly their relationship with themselves and their bodies, their relationships with others, and their participation in social and leisure time

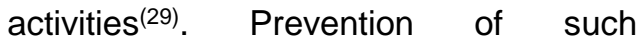
problems often requires education and health promotion strategies. The nurse can have an important role to play as a wound ostomy continence nurse ${ }^{(28)}$.

This study was carried out to develop a nursing intervention for patients with urostomy about self-care and evaluate the impact of the intervention on their compliance in practicing self-care. The study included two similar groups, a study and a control groups. Both groups were composed of patients mostly below the age of forty, predominantly males, illiterate or read and write, married, and working. Also, when patients' knowledge about urostomy was assessed before the nursing intervention, it was closely similar in both groups in almost all areas.
After implementation of the nursing intervention, the study group became more knowledgeable about the reason of urostomy performance definition, its function, related basic anatomy and physiology, compared to the control group. These differences were noticed immediately before discharge and six months after the training at the follow-up assessment. The differences were all statistically significant. Although the knowledge levels have demonstrated some slight declines at the follow-up, they remained quite high, compared to baseline levels. These results are in agreement with(7), who have also highlighted the importance of sound knowledge among urostomy patients to help them coping. They have stressed that accurate description of urostomy appearance helps ease shock at the first sight of it after ostomy surgery.

To engage in self-care activities, the individual must have the knowledge, ability, and skills to initiate and sustain 
self-care efforts. The improvement of patients' knowledge in the present study was associated with better practice of self-care activities. Thus, findings of the study highlight the significant educational role that the nurse can achieve to improve patients knowledge and practice as regards urostomy selfcare. The remarkable increase in knowledge and improvement in practice about cleaning stoma and monitoring and reporting any abnormalities among study group patients points to their urgent need for such information, which was provided to them through the nursing intervention. Hampton et al., and Patwardhan have emphasized the importance of training patients in monitoring the color, size and shape of stoma for early detection and prevention of complications. ${ }^{30,31}$

According to the present study findings, patients in the study group have retained most of their knowledge and learnt skills six months after the nursing intervention at the follow-up. The study group members were able to successfully and independently assemble all materials needed, remove the old appliance and put it, clean stoma and dry it gently, prepare the appliance and empty it correctly, and irrigate the stoma. This success might be attributed to the guidelines provided to them through individual training of patients using simple and attractive media, and simulation techniques. In this respect, Doughtily et al., have stressed the importance of teaching basic stoma care principles to enhance patients' sense of control over the situation. ${ }^{7}$

Attitudes are usually shaped by knowledge, and do influence practice. The importance of self-care as an indication of positive attempts to cope with changes has been emphasized. In the present study, statistical significant improvements were revealed in patients' attitude towards urostomy in the study group, compared to the control 
group after nursing intervention. This the quality of life for the patient by could be attributed to the role of correcting the previous serious acquiring information about the debilitating condition ${ }^{(6)}$. This was the procedure in helping patients cope with case in the present study, where both the new situation. In agreement with this, control and study groups have Hampton et al., have emphasized that patients' knowledge of structure and function increases their confidence, produces control, and reduces anxiety related to altered body function. ${ }^{30}$ Nevertheless, the attitude towards acceptance of stoma, although improved, has not reached statistical significance, compared to control group. This is quite expected, since attitudes are difficult to change, especially as regards acceptance of stoma, which undoubtedly affects the body image negatively and permanently. As mentioned by Metcalf, patient needs time to heal, to learn how to manage the urostomy, and to mentally adapt to these changes. ${ }^{32}$

Contrary to common fears of people from ostomy, it almost always improves experienced physical and/or psychological complications. However, these complications were more obvious among control group patients, especially skin complications, followed by bowel movement, then psychological disorders. These findings give support top the success of the nursing intervention in improving patients' outcomes through enhancing their knowledge, and ameliorating their attitudes and practices related to urostomy.

\section{CONCLUSION AND}

\section{RECOMMENDATIONS}

The present study underscores the significant role played by nurses as care providers for urostomy patients during their transition from the initial shock stage to acceptance of the fact that they 
can lead an active and simulating life.

The developed nursing intervention showed a remarkable and significant impact on patients' knowledge, practice and outcomes in the form of decreased complications in the study group. Therefore, it is recommended that urostomy patients should receive adequate education, training, and counseling regarding ostomy self-care during hospitalization. The nurse working with these patients must be a qualified and professional nurse to be able to deal with this sensitive and potentially depressing situation. Regular follow-up for these patients is highly recommended to keep and improve their knowledge and skills.

\section{REFERENCES}

1. Sherley E. Oncology Nursing. $2^{\text {nd }}$ ed. St. Louis: Mosby; 1999. pp. 181-186.

2. Silverberg E, Boring C, Squires T. Cancer statistics, cancer res. 37, 2828. In: Walsh $P$, Retik A, Vaughan E. Campbell's urology. $7^{\text {th }}$ ed. Philadelphia: Saunders Co; 1999. pp. 23-29.
3. Parkers S, Tong T, Colden S, Wing P. Cancer Statistics. 2001, 46(1): 452.

4. El-Sebai, M. Bladder cancer. Volume II: Cancer of the bilharzial bladder. Florida: CRC press Inc; 1983. p. 105.

5. El-balkainy N. General pathology of cancer. Cairo: Al-Asedekaa Graphics Center; 1991. P. 56.

6. Erwin R. Caring for a stoma. Nursing. 2001, 31(5): 36-45.

7. Doughtily GB, Jackson DB. Gastrointestinal disorders. St. Louis: Mosby Year Book; 2002. pp. 1-22.

8. Jean A, Edna M. Identification of stressor and use of coping methods in chronic illness. Nursing Research. 1999. 37(4): 236-9.

9. Baber S, Billing S. Adult and child care. $2^{\text {nd }}$ ed. St. Louis: Mosby Co; 2000. p. 432.

10. Perper B, Mikals C. Pre discharge and post discharge concerns of patients with an ostomy. Journal of Ostomy and Continence. 2001, 33(2): 105-9.

11. Dal J. Living with colostomy. A young persons guide. Available from: www.ostomy.fsnet.Co.Uk. 2003.

12. Fries C. Managing an ostomy. Available from: www. Nursing.Com. 2000.

13. Smeltzer S, Bare B. Urinary and renal function. Raven: Lippincott; 2000. pp. 1171-1178.

14. Saleh N, Ali A, Khalil H. Effect of psycho educational intervention on 
anxiety among Egyptian bladder cancer patients. Cancer Nursing. 2004, 12(4): 236-47.

15. Beverley F. British studies which measure patient outcome. Journal of Advanced Nursing. 1997, 26:320-8.

16. Lui, M. Chinese elderly patients' perception of their rehabilitation needs. Journal of Advanced Nursing. 1999, 30 (2): 391-400.

17. American Cancer Association. Free from smoking. Available from: HTTP://www.Ffsonline.org. 2002.

18. Flaherty $\mathbf{J}$, Davis $\mathbf{J}$, Janicak $\mathbf{P}$. Psychiatry: Diagnosis and therapy, ( $2^{\text {nd }}$ ed.). USA: Prentice-Hall International Inc; 1995. PP.474-475.

19. McMahon E. Teaching patients with acute conditions. USA: Springhouse Corporation; 1996. p. 295.

20. Abley C. Teaching elderly patients how to use inhalers. A study to evaluate an education program on inhaler technique for elderly Patients. Journal of Advanced Nursing. 1997, 25: 699-708.

21. Bulechek G, McCloskey J. Nursing interventions: Essential nursing treatments. (2 $2^{\text {nd }}$ ed.). Mexico: W.B. Saunders Company; 1998.PP. 437446.

22. The Cheshire Medical Center. Handbook of pulmonary rehabilitation program. 2000.

23. Hodgkin J, Connors G, Bell C. Pulmonary rehabilitation: guidelines to success. $2^{\text {nd }}$ ed. USA: Lippincott Company; 2000. pp. 106-110, 126128, 222-236, 496-202. Available from:

http://www.Chershire.med.com/progr ams/pulrehab/COPD

24. Linton A, Matteson M, Maebius N. Introductory nursing care of adults. $3^{\text {rd }}$ ed. USA: W.B. Saunders Company; 2000. pp. 444-503.

25. Zastocki D, Wegner C. Home care patient and family instructions. $\left(2^{\text {nd }}\right.$ ed.). USA: W.B. Saunders Comp; 2000. P.77

26. Donnell D, Aaron S, Bourbeau J. Canadian urinary society recommendations for management of urinary tract disorders. Can. Urin J. 2003, 10: 11-40.

27. Nidus Information Services. Asthma in adults. Available from: http://www.well-connceted.com. 1999.

28. Wilkines L. The basics of ostomies. Gastroenterology nursing, nursing center, library. Nursing Journal. 2003. Available from: www. Nursing center.Com/library/Journals article. 2003.

29. Secord C. Adjusting to life with an ostomy. A psycho-educational program. Canadian Nurse. 2001,7(1): 29-32.

30. Hampton B, Groenwid S, Frogge MH, Goodman M, Yarbro C. Cancer nursing, principles and practice. $4^{\text {th }}$ ed. Boston: Jones and Bartlett; 2002. 259-81.

31. Patwardhan A. An ostomy care and management total memorial hospital. Interostomal Journal. 2003, 12. 
32. Metcalf C. Practical aspects of stoma

2001,

97(12):

40-4. care. Best practice. Nursing Times. 